\title{
Demographic and Clinical Features of 1,641 Patients with Alopecia Areata, Alopecia Totalis, and Alopecia Universalis: A Single-Center Retrospective Study
}

\author{
Tugba Kevser Uzuncakmak Burhan Engin Server Serdaroglu Yalcin Tuzun
}

Department of Dermatology, Istanbul University Cerrahpasa Medical Faculty, Istanbul, Turkey

\section{Keywords}

Alopecia areata · Alopecia totalis · Alopecia universalis ·

Epidemiology

\begin{abstract}
Background/Aim: Alopecia areata ( $\mathrm{AA}$ ) is a common autoimmune hair disorder which is characterized by noncicatricial hair loss. AA commonly presents with localized patches on the scalp and face but may affect any hair-bearing region of the body leading to even more generalized involvement. AA may affect any age group, gender, and race. The current study investigates the demographic characteristics of the patients with $A A$ and subgroups of $A A$ including alopecia totalis (AT) and alopecia universalis (AU) and the prevalence of disease, sex, and age distribution and seasonal variation retrospectively in a tertiary dermatology clinic in Turkey. $\mathbf{M a -}$ terials and Methods: In this retrospective, cross-sectional study, 1,641 patients diagnosed with AA, AT, and AU in the dermatology clinic of a public university hospital were included. The dermatology outpatient database was reviewed retrospectively. The diagnosis of AA was based on patient history, clinical examinations, and histopathologic findings. Results: Fifty-four thousand one hundred sixty-eight patients were admitted to our outpatient clinic in 4 years time,
\end{abstract}

and 1,641 were diagnosed as having AA, AT, and AU. One thousand three hundred ninety-two patients $(84.8 \%)$ had AA, 81 (4.9\%) had AT, and 168 (10.2\%) had AU. Among the 1,641 patients included in the study, 877 were females (53.4\%) and 764 were males (46.6\%). The mean age was $29.86 \pm 14.48$ years in AA, $29.50 \pm 16.18$ in AT, and $32.81 \pm$ 14.48 in $A U ; 77.4,72.8$, and $68.5 \%$ of patients were aged under 40 years in AA, AT, and AU. There was no statistically significant difference in seasonal presentation times. Conclusion: $A A$ is affecting approximately $2 \%$ of the general population without any sex, race, or age group predilection. In this study, we found a lower prevalence of AA in the pediatric age group in comparison with adults. This finding may support the hypothesis of the increasing prevalence of AA over time. The higher ratio of AA regarding this study may support that the frequency of $A A$ and subtypes varies between regions.

(c) 2020 S. Karger AG, Basel

\section{Introduction}

Alopecia areata (AA) is a T-cell mediated, autoimmune disorder which is mainly affecting anagen hair follicles and nails. Clinically, AA usually presents with localized circumscribed patches of nonscarring hair loss [1].

$\begin{aligned} & \text { karger@karger.com } \\ & \text { www.karger.com/sad }\end{aligned}$
Karger ${ }^{\prime /}$

Tugba Kevser Uzuncakmak

Department of Dermatology, Istanbul University Cerrahpasa Medical Faculty Dr. Erkin St.

Istanbul 34722 (Turkey)

tkevserustunbas@gmail.com 
Table 1. Sex distribution of patients with AA, AT, and AU according to age

\begin{tabular}{|c|c|c|c|c|c|c|c|c|c|}
\hline \multirow[t]{3}{*}{ Age groups } & \multicolumn{9}{|l|}{ Sex } \\
\hline & \multicolumn{3}{|l|}{$\mathrm{AA}$} & \multicolumn{3}{|l|}{ AT } & \multicolumn{3}{|l|}{$\mathrm{AU}$} \\
\hline & male & female & total & male & female & total & male & female & total \\
\hline $0-9$ & 41 & 43 & 84 & 5 & 2 & 7 & 0 & 4 & 4 \\
\hline$\%$ sex & 6.4 & 5.7 & 6 & 15.6 & 4.1 & 8.6 & 0.0 & 5.0 & 2.4 \\
\hline 10-19 & 149 & 122 & 271 & 8 & 9 & 17 & 16 & 10 & 26 \\
\hline$\%$ sex & 23.1 & 16.3 & 19.5 & 25.0 & 18.4 & 21.0 & 18.2 & 12.5 & 15.5 \\
\hline $20-29$ & 158 & 226 & 384 & 10 & 17 & 27 & 33 & 15 & 48 \\
\hline$\%$ sex & 24.5 & 30.2 & 27.6 & 31.3 & 34.7 & 33.3 & 37.5 & 18.8 & 28.6 \\
\hline 30-39 & 173 & 165 & 338 & 2 & 6 & 8 & 19 & 18 & 37 \\
\hline$\%$ sex & 26.9 & 22.1 & 24.3 & 6.3 & 12.2 & 9.9 & 21.6 & 22.5 & 22.0 \\
\hline $40-49$ & 79 & 98 & 177 & 5 & 7 & 12 & 12 & 20 & 32 \\
\hline$\%$ sex & 12.3 & 13.1 & 12.7 & 15.6 & 14.3 & 14.8 & 13.6 & 25.0 & 19.0 \\
\hline 50-59 & 42 & 81 & 123 & 1 & 8 & 9 & 8 & 11 & 19 \\
\hline$\%$ sex & 6.5 & 10.8 & 8.8 & 3.1 & 16.3 & 11.1 & 9.1 & 13.8 & 11.3 \\
\hline$>60$ & 2 & 13 & 15 & 1 & 0 & 1 & 0 & 2 & 2 \\
\hline$\%$ sex & 0.3 & 1.7 & 1.1 & 3.1 & 0.0 & 1.2 & 0.0 & 2.5 & 1.2 \\
\hline Total & 644 & 748 & 1,392 & 32 & 49 & 81 & 88 & 80 & 168 \\
\hline$\% \operatorname{sex}$ & 100.0 & 100.0 & 100.0 & 100.0 & 100.0 & 100.0 & 100.0 & 100.0 & 100.0 \\
\hline
\end{tabular}

AA, alopecia areata; AT, alopecia totalis; AU, alopecia universalis.

Alopecia totalis (AT) is a subtype of AA which is characterized by total hair loss on the face and scalp, whereas alopecia universalis (AU) is the other subtype of AA manifesting with generalized hair loss on hair-bearing regions of the body. AT and AU usually have poorer prognosis than AA itself. In the literature, there are very few studies about the clinical epidemiology of AA. In hospital-based studies, the incidence of AA was found to range from 0.57 to $3.8 \%$, and in general population studies, the lifetime incidence of AA was reported to be $1.7-2.1 \%$ and prevalence was $0.1-0.2 \%[2,3]$. On the other hand, it is still debated that AA prevalence may differ by age or other demographic and clinical characteristics. The goal of this study was to determine the differences of the demographic characteristics of the patients with AA, AT, and AU, including the prevalence of disease, sex, and age distribution and seasonal variation retrospectively in a tertiary referral center in Turkey.

\section{Materials and Methods}

This retrospective, cross-sectional study was conducted in the dermatology clinic of a public university hospital. One thousand six hundred forty-one patients diagnosed with AA, AT, and AU were investigated. Diagnosis of AA was based on patient history, clinical examinations, and histopathologic findings.

Alopecia Areata and Demographic Characteristics

\section{Statistical Analysis}

Data analysis was performed using the NCSS statistical package 2007 (Kaysville, UT, USA). The results were analyzed by using percentile values and $\chi^{2}$ tests. Statistical significance was set at the $0.5 \%$ level.

\section{Results}

\section{Clinical Characteristics and Patterns of $A A$}

Of 54,168 dermatology outpatient clinic patients, 1,641 were diagnosed as having AA (3.02\%), 1,392 had patchy AA (84.8\%), 81 had AT (4.9\%), and 168 had AU $(10.2 \%)$. For patients who made recurrent presentations, the first visit date was used. For all subtypes of AA, prevalence, age, and sex distribution and presentation times were recorded and analyzed according to disease subtype. Treatment modalities were not analyzed in this study.

\section{Sex Distribution}

Of the total 1,641 patients with alopecia, 877 (53.4\%) of the patients were female and 764 (46.6\%) were male, with a female to male ratio of 1.14:1. There were no statistically significant differences between AA, AT, and AU groups ( $p=0.138)$. Of the 1,392 patients with AA, 644 


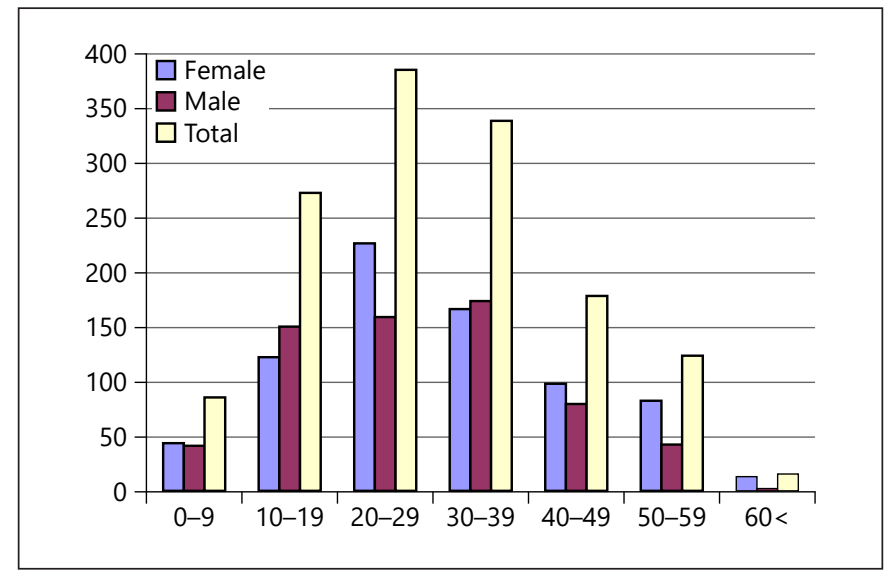

Fig. 1. Sex distribution of patients with AA according to age. AA, alopecia areata.

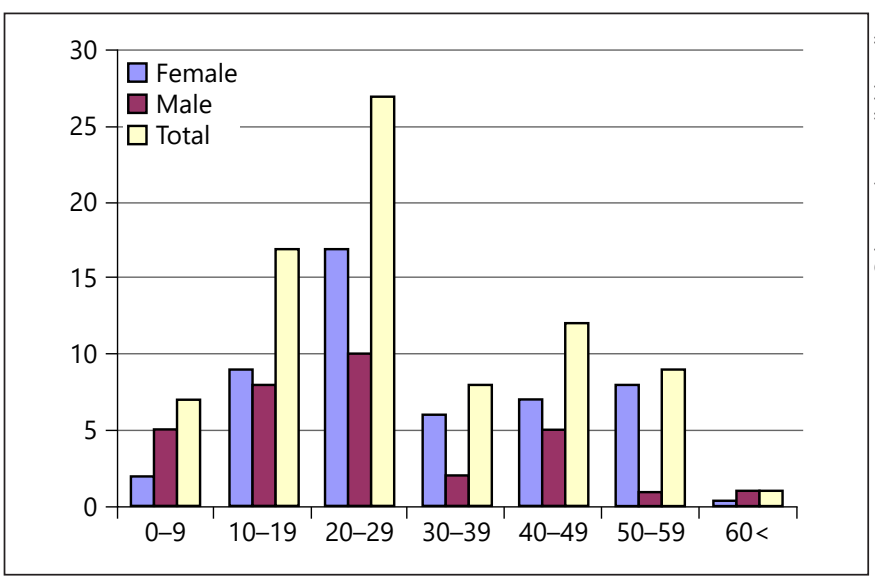

Fig. 2. Sex distribution of patients with AT according to age. AT, alopecia totalis.

(46.3\%) were male and $748(53.7 \%)$ were female, with a female to male ratio of 1.15:1. Eighty-one (4.9\%) patients were diagnosed as having AT, 32 (39.5\%) of whom were male and $49(60.5 \%)$ were female, with a female to male ratio of 1.53:1. One hundred sixty-eight (10.2\%) patients were diagnosed as having AU, 88 (52.4\%) of whom were male and $80(47.6 \%)$ were female, with a female to male ratio of 1:1.10.

\section{Age Distribution}

The mean age of patients with AA was $29.86 \pm 14.48$ (range: $3-96$ ) years. Patients were classified into 7 groups according to age as $0-9,10-19,20-29,30-39,40-49,50-$ 59 , and $>60$ years (Table 1 ). AA was most common in the 30 - to 39 -year age group among men and the 20- to 29-

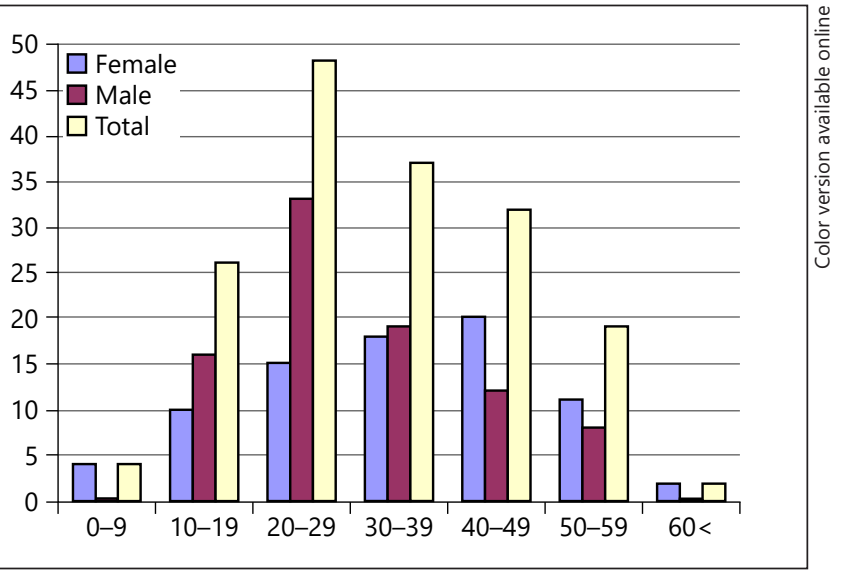

Fig. 3. Sex distribution of patients with AU according to age. AU, alopecia universalis.

Table 2. Seasonal variation of AA, AT, and AU

\begin{tabular}{lllll}
\hline & Winter & Spring & Summer & Autumn \\
\hline AA, \% & 30.7 & 26.4 & 20.2 & 22.7 \\
AT, \% & 32.1 & 28.4 & 24.7 & 14.8 \\
AU, \% & 27.4 & 29.2 & 23.8 & 19.6 \\
\hline
\end{tabular}

AA, alopecia areata; AT, alopecia totalis; AU, alopecia universalis.

year age group among women. Three-quarters (77.4\%) of the patients with AA were aged younger than 40 years, and only $1.1 \%$ were aged over 60 years. AA was more common in males in the pediatric age group ( $0-19$ years), whereas it was more common in females in adults. In the geriatric age group, AA was more common in females (Table 1; Fig. 1).

The median age of the patients with AT was $29.50 \pm$ 16.18 (range: $3-84$ ) years. The disease was most commonly seen in the 20- to 29 -year age group in both sex. Some $2.8 \%$ of the patients were aged younger than 40 years, and $1.2 \%$ were aged over 60 years. AT was more common in males both in the pediatric and the geriatric age groups (Table 1; Fig. 2).

The median age of patients with AU was $32.81 \pm 14.48$ (range: 4-77) years. The disease was more common in the 20 - to 29-year age group in males and in the 40 - to 49 -year age group in females. Some $17.9 \%$ of the patients were in the pediatric age group, and $1.2 \%$ were aged over 60 years. AU was more common in females both in the pediatric and the geriatric age groups (Table 1; Fig. 3).
10

Skin Appendage Disord 2021;7:8-12 DOI: $10.1159 / 000510880$ 


\section{Presentation Times}

The seasonal variation of AA, AT, and AU is summarized in Table 2 . There were no statistically significant differences in the presentation times between all groups $(p>0.05)$.

\section{Discussion}

AA is a common noncicatricial hair disorder that negatively impacts the health-related quality of life of children and adults. AA may affect both sex and may occur at any age. Unfortunately, almost $10-15 \%$ of patients with AA are predicted to progress into complete scalp (AT) or scalp and body (AU) hair loss forms [4, 5].

In previous observational studies, the global prevalence of AA was reported to be approximately $2 \%$, with significant regional variability [2]. In our study, the prevalence of AA was found as $3.02 \%$ of all dermatoses. We think that the prevalence of AA may be increasing over time. Being a referral center of our clinic also may affect this high prevalence ratio.

In the literature, it was reported that most of the $\mathrm{AA}$ patients have limited-patchy lesions on the face and scalp, 14-25\% have AT, and less than $10 \%$ have AU [5]. Similarly, in our study, $84.8 \%$ of patients had patchy AA, $4.9 \%$ had AT, and $10.2 \%$ had AU.

AA may affect both women and men [5-7]. In most of the observational studies, AA has been reported to be more common in females; however, there are few studies in which AA was reported to be more common in males [8-16]. In this study, we found female predominance in patients with AA and AT. This may also support the general female predominance in autoimmune disorders [17]. AU was more common in males in this study. Male gender was reported to be one of the poor diagnosis criteria of AA in the literature [7].

In the literature, AA was reported to be more common in the younger age groups of children/adolescents than adults overall $[2,18]$. In our study, prevalence of AA was higher in adults than the pediatric age group. Almost $25.5 \%$ of the patients with AA, $29.6 \%$ of AT, and $17.9 \%$ of $\mathrm{AU}$ were in the pediatric age group. Presentation of pediatric patients to general practitioners or pediatricians may affect these ratios.

In adults, AA was reported to be more common in patients aged under 40 years. Similarly, in our study, 77.4\% of patients with AA, $72.8 \%$ of those with AT, and $68.5 \%$ of patients with $\mathrm{AU}$ were younger than 40 years of age. AA was more common in female patients aged under 40

Alopecia Areata and Demographic

Characteristics years. AT and AU were more common in male patients younger than 40 years. In our study, all AA subtypes were more common in male patients aged under 20 years. These findings may support the hypothesis of the worse progression of AA in male patients, as reported in the literature $[9,17]$.

In the literature, Seyrafi et al. [10] reported the median age of patients with AA as $24.05 \pm 9.98$ and Kavak et al. [7] reported it as $24.32 \pm 0.54$ years [7, 18]. In our study, AA, AT, and AU were most commonly seen in the 20 - to 29 -year age group with a median age of $29.86 \pm 14.48$ in AA, $29.50 \pm 16.18$ in AT, and $32.81 \pm 14.48$ in AU. The higher median age of AA may be related to the progressive course of AA to AU by time.

In our study, when we compared the seasonal presentation times of the patients, although there were no statistically significant differences, AA and AT patients were mostly admitted in winter and AU patients were admitted in spring. In the literature, there are few studies about seasonal application time, and no seasonal difference was reported in AA; yet in some studies, disease activity was reported to increase during winter $[11,16]$. We think that the increased prevalence of AA and AT during winter may be related to the triggering role of psychological distress in AA pathogenesis. The role of stressful events such as school and work life may have an extra role in disease activity. Also, seasonal activity has been reported in atopic patients with AA in the literature $[2,15,19]$. This coexistence may also have a role in the worsening of both atopic dermatitis and AA during the winter time.

\section{Limitations}

Limitations of our study include its retrospective nature and the fact that in many cases skin physical exam findings or a treatment plan have not been recorded.

\section{Conclusion}

AA is a one of the skin disorders that is garnering attention due to research about novel treatment modalities and pathogenesis. As the global prevalence of AA is estimated to be approximately $2 \%$, it appears to be increasing over time, as found in this study. We would like to contribute to the literature with epidemiologic data.

\section{Acknowledgements}

We would like to thank our Department of Statistics, in particular Hayriye Vehid, MD. 


\section{Statement of Ethics}

The study was carried out in accordance with the World Medical Association Declaration of Helsinki and was approved by the local ethics committee.

\section{Conflict of Interest Statement}

The authors have no conflicts of interest to disclose.

\section{Funding Sources}

The authors did not receive any funding.

\section{Author Contributions}

Conception and design of the study: Tugba Kevser Uzuncakmak and Burhan Engin; acquisition of data: Tugba Kevser Uzuncakmak and Server Serdaroğlu; analysis and/or interpretation of data:Tugba Kevser Uzuncakmak, Burhan Engin, Server Serdaroğlu, and Yalçın Tuzun.

\section{References}

1 Thompson JM, Park MK, Qureshi AA, Cho E. Race and alopecia areata amongst US women. J Investig Dermatol Symp Proc. 2018;19:4750.

2 Lee HH, Gwillim E, Patel KR, Hua T, Rastogi $S$, Ibler E, et al. Epidemiology of alopecia areata, ophiasis, totalis and universalis: a systematic review and meta-analysis. J Am Acad Dermatol. 2020 Mar;82(3):675-82.

3 Richard MA, Corgibet F, Beylot-Barry M, Barbaud A, Bodemer C, Chaussade V, et al. Sex- and age-adjusted prevalence estimates of five chronic inflammatory skin diseases in France: results of the "OBJECTIFS PEAU" study. J Eur Acad Dermatol Venereol. 2018 Mar 23;32(11):1967-71.

4 Tosti A, Bellavista S, Iorizzo M. Alopecia areata: a long term follow-up study of $191 \mathrm{pa}-$ tients. J Am Acad Dermatol. 2006;55:438-41.

5 Messenger AG, McKillop J, Farrant P, McDonagh AJ, Sladden M. British Association of Dermatologists' guidelines for the management of alopecia areata 2012. Br J Dermatol. 2012;166(5):916-26.

6 Safavi KH, Muller SA, Suman VJ, Moshell AN, Melton LJ. Incidence of alopecia areata in
Olmsted County, Minnesota, 1975 through 1989. Mayo Clin Proc. 1995;70(7):628-33.

7 Kavak A, Yeşildal N, Parlak AH, Gökdemir G, Aydoğan I, Anul H, et al. Alopecia areata in Turkey: demographic and clinical features. J Eur Acad Dermatol Venereol. 2008;22(8): 977-81.

8 Kyriakis KP, Paltatzidou K, Kosma E, Sofouri E, Tadros A, Rachioti E. Alopecia areata prevalence by gender and age. J Eur Acad Dermatol Venereol. 2009;23(5):572-3.

9 Tan E, Tay YK, Goh CL, Chin Giam Y. The pattern and profile of alopecia areata in Singapore: a study of 219 Asians. Int J Dermatol. 2002;41(11):748-53.

10 Seyrafi H, Akhiani M, Abbasi H, Mirpour S, Gholamrezanezhad A. Evaluation of the profile of alopecia areata and the prevalence of thyroid function test abnormalities and serum autoantibodies in Iranian patients. BMC Dermatol. 2005;5:11.

11 Ro BI. Alopecia areata in Korea (1982-1994). J Dermatol. 1995;22(11):858-64.

12 Goh C, Finkel M, Christos PJ, Sinha AA. Profile of 513 patients with alopecia areata: associations of disease subtypes with atopy, auto- immune disease and positive family history. J Eur Acad Dermatol Venereol. 2006;20(9): 1055-60.

13 Wang E, McElwee KJ. Etiopathogenesis of alopecia areata: why do our patients get it? Dermatol Ther. 2011;24(3):337-47.

14 Dainichi T, Kabashima K. Alopecia areata: what's new in epidemiology, pathogenesis, diagnosis, and therapeutic options? J Dermatol Sci. 2017;86(1):3-12.

15 Crosby DL, Gammon WR. Seasonal alopecia areata with atopy. J Am Acad Dermatol. 1989; 21(4 Pt 1):806-7.

16 Fiedler VC, Alaiti S. Treatment of alopecia areata. Dermatol Clin. 1996;14(4):733-7.

17 Fairweather D, Frisancho-Kiss S, Rose NR. Sex differences in autoimmune disease from a pathological perspective. Am J Pathol. 2008; 173(3):600-9.

18 Fricke ACV, Miteva M. Epidemiology and burden of alopecia areata: a systematic review. Clin Cosmet Investig Dermatol. 2015;8:397403.

19 Putterman E, Castelo-Soccio L. Seasonal patterns in alopecia areata, totalis, and universalis. J Am Acad Dermatol. 2018;79(5):974-5. 